\title{
Prognostic value of pro-inflammatory neutrophils and C-reactive protein in cancer patient with COVID- 19: a multi-center, retrospective study
}

\section{Bo Zhang}

Wuhan Union Hospital

Yuanhang Yu

Wuhan Union Hospital

Shawna Herbet

University of Texas MD Anderson Cancer Center

Yue Zhang

Wuhan Union Hospital

Jianhua Lu

Chibi People's Hospital

Shihua Liu

Zaoyang First People's Hospital

Fang Xie

Ezhou Central Hospital

Liang Zhao

Huangshi Central Hospital

Xiao Lei

Wuhan Union Hospital

Wei Deng

Wuhan Union Hospital

Jianying Chen

Wuhan Union Hospital

Yunqiao Li (D liyunqiao@hust.edu.cn )

\section{Research}

Keywords: Cancer, COVID-19, Pro-inflammatory, Neutrophils, C-reactive protein

Posted Date: July 2nd, 2020

DOI: https://doi.org/10.21203/rs.3.rs-37464/v1 
License: (c) (i) This work is licensed under a Creative Commons Attribution 4.0 International License. Read Full License 

in cancer patient with COVID-19: a multi-center, retrospective study

3 Bo Zhang ${ }^{1 *}$, Yuanhang Yu ${ }^{1 *}$, Shawna Herbet ${ }^{2}$, Yue Zhang ${ }^{1}$, Jianhua Lu $^{3}$, Shihua Liu ${ }^{4}$,

4 Fang $\mathrm{Xie}^{5}$, Liang $\mathrm{Zhao}^{6}$, Xiao Lei ${ }^{7}$, Wei Deng ${ }^{7}$, Jianying Chen ${ }^{8 \#}$, Yunqiao $\mathrm{Li}^{\text {9\# }}$

5 1. Department of Thyroid and Breast Surgery, Union Hospital, Tongji Medical

6 College, Huazhong University of Science and Technology, Wuhan 430022, China.

7 2. Department of Thoracic Medical Oncology; Department of Genomic Medicine, the

8 University of Texas, MD Anderson Cancer Center, Houston, TX, 77030.

9 3. Department of General Surgery, Chibi People’s Hospital, Chibi, China.

10 4. Department of Infectious Diseases, Zaoyang First People’s Hospital, Zaoyang,

11 China.

12 5. Department of Respiratory Intensive Care Unit, Ezhou Central Hospital, Ezhou,

13 China.

14 6. Department of General Surgery, Huangshi Central Hospital, Huangshi, China.

15 7. Department of General Medicine, Union Hospital, Tongji Medical College,

16 Huazhong University of Science and Technology, Wuhan 430022, China. 
8. Department of Gastrointestinal Surgery, Union Hospital, Tongji Medical College,

18 Huazhong University of Science and Technology, Wuhan 430022, China.

19 9. Department of Geriatrics, Union Hospital, Tongji Medical College, Huazhong

20 University of Science and Technology, Wuhan 430022, China.

$21 *$ Bo. Zhang and Yuanhang Yu contributed equally to this study.

22 \# Corresponding Author

23 Yunqiao Li, Department of Geriatrics, Union Hospital, Tongji Medical College,

24 Huazhong University of Science and Technology, 1277 Jiefang Road, Wuhan 430022 ,

25 China, Email: liyunqiao@hust.edu.cn.

26 Jianying Chen, Department of Gastrointestinal Surgery, Union Hospital, Tongji

27 Medical College, Huazhong University of Science and Technology, Wuhan 430022,

28 China, Email: bobytail@sina.com. 
33 Abstracts

34 Background: At present, the epidemic of the novel coronavirus disease 2019

35 (COVID-19) has quickly engulfed the world. Inflammatory cytokines are associated with the severity and outcomes of patients with COVID-19. However, the effects of pro-inflammatory factors in cancer patients with COVID-19 are unknown.

Methods: A multi-center, retrospective, cross-sectional study, based on 5 designated tertiary hospitals for the treatment of COVID-19 in Hubei Province, China. 112 cancer patients with COVID-19, and 105 COVID-19 patients without cancer were

41 enrolled in the study between January $1^{\text {st }}, 2020$ and April $30^{\text {th }}, 2020$. The risk assessment of pro-inflammatory factors for disease severity and clinical adverse outcomes was identified by univariable and multivariable logistic regression models.

44 Results: Of the 112 cancer patients with COVID-19, 40 (35.7\%) patients were in

45 critical condition and 18 (16.1\%) patients died unfortunately. Univariate and multivariate analysis demonstrated that hemoglobin count and pro-inflammatory neutrophil and C-reactive protein, can be used as independent factors affecting the severity of COVID-19; Meanwhile, pro-inflammatory neutrophils and C-reactive 
49 protein can be used as an independent influencing factor for adverse clinical outcome.

50 Moreover, the dynamic changes of neutrophils and C-reactive protein were also

51 presented, and compared with COVID-19 patients without cancer, cancer patients

52 with COVID-19 showed higher neutrophil counts and C-reactive protein levels.

53 Conclusions: In cancer patients with COVID-19, the significant increase in pro-

54 inflammatory neutrophil and C-reactive protein indicated a more critical illness and

55 adverse clinical outcome, and pro-inflammatory neutrophils and C-reactive protein

56 played a more adverse effect compare with COVID-19 patients without cancer, which

57 may be the cause of critical illness and adverse clinical outcomes of cancer patients

58 with COVID-19.

59 Keywords: Cancer; COVID-19; Pro-inflammatory; Neutrophils; C-reactive protein.

60 


\section{Background}

Since the outbreak of COVID-19 in Wuhan in 2019, the outbreak of COVID-19 has

quickly spread to Hubei Province and quickly engulfed China $(1,2)$. Now the

epidemic has rapidly spread to the worldwide. As of May 16, 2020, COVID-19 had spread to 188 countries around the world, which caused more than 4.5 million people being infected, and more than 300,000 people died. The most common manifestations

71 of COVID-19 infected patients include fever, cough, fatigue, dyspnea, and radiological evidence of pneumonia (3). Its mean incubation period is 5.2 days (4).

73 COVID-19 is directly transmitted through respiratory droplets or indirectly through

74 pollutants and be able to secondary transmit within the family (5).

75 While facing the epidemic, cancer is still a major disease. According to reports,

76 there were more than 4.2 million new cancer cases in China in 2015 (6), and 1.7

77 billion new cancer cases in the United States in 2019 (7). To data, studies have

78 reported that during this epidemic, cancer patients are more susceptible to COVID-19

$79(8,9)$. However, their research was limited to the study of small samples and solid 
tumors, lack of laboratory data, and insufficient evidence to support relevant

81 conclusions $(10,11)$.

82 Moreover, studies have reported that pro-inflammatory factors such as CRP,

83 interleukin 6, neutrophils, etc. were associated with the severity and prognosis of

84 patients with COVID-19 (12-14). In patients with COVID-19, high levels of pro-

85 inflammatory factors predicted a poor prognosis. However, whether inflammatory

86 factors play the same adverse effect in cancer patients with COVID-19 is still unclear.

87 In this study, we collected medical data of 112 cancer patients from 5 designated

88 tertiary hospitals for the treatment of COVID-19 in Hubei Province, where the

89 prevalence of COVID-19 was the earliest and most severely in China. We aim to

90 analyze the laboratory data and outcomes of patients through retrospective research,

91 and then find out the risk correlation of pro-inflammatory factors related to the

92 clinical severity and outcomes of cancer patients with COVID-19. Deepen the

93 understanding of the impact of the COVID-19 on cancer patients, demonstrate the

94 adverse effects of pro-inflammatory factors in cancer patients with COVID-19, and

95 provide help for the treatment of cancer patients. 


\section{Study design, setting and participants}

98 This was a retrospective, cross-sectional, multicenter study, which was performed in 5

99 designated tertiary hospitals for the treatment of COVID-19 in Hubei, China. These

100 data were from 112 cancer patients infected with COVID-19, and 105 COVID-19

101 patients without cancer, who were enrolled from January $1^{\text {st }}$ to April $30^{\text {th }}$

102 2020. Clinical retrospective data was retrieved from the medical records, including

103 demographic information, underlying comorbidities, chest computed tomographic

104 (CT) scans, laboratory data, anti-tumor therapy, and outcomes. The clinical outcomes

105 of these cancer patients were monitored up to April 30, 2020, the final date of follow-

106 up, when all the patients were discharged. All data were reviewed by two physicians

107 respectively to verify data accuracy. COVID-19 patients without cancer were

108 randomly selected. This study was reviewed and approved by Ethics Committee of

109 the Tongji Medical College of Huazhong University of Science and Technology

110 (No.TJ-2020S098). The exemption from informed patients' consent was approved by 
111 the ethics committee because of the rapid spread of the infection and the rapid

112 progression of some cases.

\section{Definitions}

114 COVID-19 pneumonia was diagnosed according to the updated COVID-19

115 Diagnostic Criteria, and clinical diagnostic criteria and laboratory nucleic acid

116 detection confirmation criteria by RT-PCR of nasal and/or pharyngeal specimens. The

117 patient's clinical condition is diagnosed according to Guidelines for the Diagnosis and

118 Treatment of Novel Coronavirus (2019-nCoV) Infection by the National Health

119 Commission of the People's Republic of China (Trial Version 7). The evaluation

120 indicators of clinical outcomes are survival and death.

\section{Statistical methods}

122 We fitted 2 types of models. For univariate analysis, categorical variables were

123 expressed as frequency rates and percentages and compared by using Pearson's chi-

124 square, although the Fisher's exact test was used when the data were limited, and by

125 using Mann-Whitney test. Continuous variables were described using mean, median,

126 and interquartile range (IQR) values and compared by using independent group t tests 

used. In addition, in multivariate analysis, binary logistic regression analysis is used

129 for binary classification variables such as survival status; ordinal logistic regression

130 analysis is used for ordered classification variables such as disease severity. All

131 statistical analyses were performed with the SPSS (version 23.0) software.

\section{Results}

133 Presenting demographic, Clinical Characteristics and Outcome

134 A total of 112 cancers infected with COVID-19 (Supplementary Table 1), the median

135 age is 62.95 years old (SD, 15.70), $60(53.6 \%)$ patients are male, $22(19.6 \%)$ patients

136 were non-solid tumor patients. Lung cancer $(23,20.5 \%)$ was the most frequent

137 type, following by breast cancer $(11,9.8 \%)$ and leukemia $(11,9.8 \%)$, hypertension

$138(33,29.5 \%)$ was the most common comorbidity, and $27(24.1 \%)$ patients with

139 multiple comorbidities. $12(10.7 \%)$ patients received anti-tumor therapy with

140 chemotherapy within 28 days before diagnosis of COVID-19, 58 (51.8\%) patients

141 developed severe condition, 40 (35.1\%) patients developed critical condition, and 18

142 (16.1\%) patients died unfortunately. 
144 Relative to COIVD-19 patients without cancer, cancer patients with COVID-19 show

145 a higher risk of critical illness. Therefore, we analyzed the laboratory parameters of

146 cancer patients with COVID-19 on admission (Supplementary Table 2). Relative to

147 the normal reference value range and COIVD-19 patients without cancer, cancer

148 patients with COVID-19 showed a lower erythrocyte count, $\times 10^{9} / \mathrm{L}$ (3.88, IQR [3.21-

149 4.09]) and hemoglobin count, $\times 10^{9} / \mathrm{L}(114.00$, IQR [102.00-273.50]), a higher

150 neutrophil count, $\times 10^{9} / \mathrm{L}(3.95$, IQR [2.73-6.04]) and higher levels of CRP, mg/L

151 (35.10, IQR [5.68-81.90]).

152 Independent factors for the severity of COVID-19

153 Further, we analyzed the correlation between inflammatory factors in laboratory

154 parameters and the severity of COVID-19. Univariate analysis showed that the

155 erythrocyte count, hemoglobin count, neutrophil count, lactate dehydrogenase, CK-

$156 \mathrm{MB}$, troponin I, procalcitonin, CRP, IL-6, and IL-10 may be factors that affect the

157 severity of COVID-19 (Table 1). Compared with non-critically condition patients,

158 critically illness patients showed lower erythrocyte count and hemoglobin count, 
higher neutrophil count and higher levels of LDH, CK-MB, troponin I, procalcitonin,

CRP, IL-6, and IL-10. Moreover, Multivariate analysis showed that hemoglobin

counts (OR, 0.89; 95\% CI, 0.81-0.98; $P=0.022)$, neutrophil counts (OR, 1.80; 95\%

CI, 1.09-2.97; $P=0.021)$, and CRP (OR, $1.04 ; 95 \%$ CI, $1.001-1.071 ; P=0.043)$

163 levels were found to be independent predictors of COVID-19 severity (Table 2).

164 Lower hemoglobin number, higher neutrophil count, and higher CRP levels were

165 predictive of critical condition.

Independent factors for the adverse clinical outcome of COVID-19

Meanwhile, we analyzed the correlation between inflammatory factors in laboratory

parameters and the adverse clinical outcomes of COVID-19. Univariate analysis

169 showed that erythrocyte count, hemoglobin count, neutrophil count, LDH,

170 procalcitonin, CRP, and IL-6 may be factors that affect the adverse clinical outcome

171 of COVID-19 (Table 3). Compared to surviving patients, non-surviving patients also

172 showed lower erythrocyte count and hemoglobin count, higher neutrophil counts, and

173 higher levels of LDH, procalcitonin, CRP, and IL-6. Multivariate analysis showed

174 that neutrophil counts (OR, 1.286; 95\% CI, 1.007-1.536; $P=0.005)$ and CRP (OR, 

predictors of adverse clinical outcomes of COVID-19 (Table 4). Higher neutrophil

177 counts and higher levels of CRP were predictive of adverse clinical outcomes.

\section{Dynamic changes of pro-inflammatory neutrophils and CRP}

179 The above results indicated that the pro-inflammatory neutrophils and CRP are

180 independent predictors of COVID-19 severity and clinical adverse outcomes.

181 Therefore, we analyzed the continuous dynamic changes of neutrophil counts and

182 CRP levels in surviving and non-surviving patients in critical condition. This dynamic

183 process of change was from admission to discharge or death. The neutrophil count

184 showed an overall higher level and an upward trend in the dynamic changes of non-

185 surviving patients, but it showed an overall lower level and a downward trend in the

186 dynamic changes of surviving patients (Figure 1A). Meanwhile, CRP showed an

187 overall higher level and an upward trend in the dynamic changes of non-surviving

188 patients, but showed an overall lower level and a downward trend in the dynamic

189 changes of surviving patients (Figure 1B). This further identified the inflammatory 
191 COVID-19.

192 Clinical factors of severity and adverse clinical outcomes

193 Studies have reported that cancer patients with non-solid tumors, comorbidities and

194 chemotherapy after infection with COVID-19 are more critically ill or have a higher

195 mortality rate. Therefore, we analyzed the correlation of clinical factors other than

196 laboratory parameters with COVID-19 severity and adverse clinical outcomes in 112

197 cancer patients with COVID-19 to verify the reliability of our data. The results

198 showed that patients with non-solid tumor cancer and myeloma were more critical

199 illness after infection with COVID-19 (Supplementary Figure 1A-B). Patients with

200 thyroid cancer were milder after infection with COVID-19 (Supplementary Figure

201 1C). Cancer patients with anemia, pleural effusion, multiple complications, and

202 chemotherapy were more critical illness after infection with COVID-19

203 (Supplementary Figure 1D-F). Moreover, gastric cancer patients, cancer patients with

204 multiple comorbidities and chemotherapy had a higher mortality rate after infection

205 with COVID-19 (Supplementary Figure 1H-J). Compared with COVID-19 patients 
without cancer, cancer patients with COVID-19 were more critical illness and had a

higher mortality (Supplementary Table 3).

\section{Discussion}

209 In this study, we retrospectively analyzed the laboratory data and clinical outcomes of

210112 cancer patients with COVID-19 and compared 105 patients with COVID-19

211 patients without cancer as controls. Our data demonstrated that although univariate

212 analysis indicate that the levels of pro-inflammatory neutrophils, procalcitonin, CRP,

213 and IL-6 are associated with the severity and adverse clinical outcomes of COVID-19,

214 multivariate analysis indicate that only neutrophils and CRP can be used as

215 independent predictors of disease severity and adverse clinical outcomes. Moreover,

216 the dynamic changes of pro-inflammatory neutrophils and CRP indicated that the

217 neutrophils count and the level of CRP in non-surviving patients remain at an overall

218 higher level and gradually increase, and compared with COVID-19 patients without

219 cancer, cancer patients with COVID-19 showed a higher neutrophil count and higher

220 levels of CRP, meanwhile showed more critical illness and higher mortality rate. 
221 Meanwhile, studies have reported that patients infected with COVID-19 show

222 increased levels of pro-inflammatory neutrophils, procalcitonin, CRP and IL-6 (15).

223 Moreover, studies have reported that the neutrophil count, IL-6 and CRP can be used

224 as predictors of clinical outcome and disease severity $(12,16,17)$. Recently, some

225 studies have shown that cancer patients with COVID-19 have higher level of CRP,

226 and higher neutrophil counts, D-dimer and $\mathrm{LDH}$ are associated with poor prognosis

$227(9,18)$. Our data also demonstrated that the expression of pro-inflammatory

228 neutrophils and C-reactive protein was significantly increased in cancer patients with

229 COVID-19, and further through multivariate regression analysis showed that they can

230 be used as independent predictors of the severity and the clinical adverse outcome of

231 COVID-19.

232 In addition, many studies have reported that cancer patients become more severe

233 and have a poor prognosis after being infected with COVID-19 (18, 19). Recent

234 research on cancer patients with COVID-19 had shown that non-surviving patients

235 exhibit higher neutrophil counts and C-reactive protein levels (20). However, the

236 correlation between the dynamic changes of cytokine levels and the prognosis of 
237 patients during disease has not been studied. Therefore, while discovering the same

238 results, we further analyzed the dynamic changes of pro-inflammatory neutrophils and

239 CRP, and further verified that the pro-inflammatory neutrophils and C-reactive

240 protein were adverse effects in cancer patients with COVID-19.

241 Meanwhile, studies have reported that inflammation promotes tumorigenesis and

242 development at all stages, and tumors can also promote the production of

243 inflammatory cells and inflammatory mediators (21). Inflammation could activate

244 neutrophils and their extracellular trap formation to promote tumor development (22).

245 Tumor development and progression can also induce inflammation. Neutrophils and

246 CRP are inflammatory marker. Elevated levels of neutrophil counts and CRP may

247 also play a causal role in the pathogenesis of cancer (23). Moreover, COVID-19 as an

248 acute inflammatory disease. Due to coronavirus infection, monocytes and

249 macrophages secrete large amounts of inflammatory cytokines and inflammatory

250 mediators such as CRP and IL-6. This can trigger cytokine release syndrome, which

251 causes ARDS to cause death (14). Neutrophils can cause neutrophil extracellular traps

252 (NETs), leading to organ damage and mortality in COVID-19 (24). 
253 Therefore, higher neutrophil counts and CRP levels in cancer patients with COVID-

25419 may be the result of the combined effect of cancer and COVID-19, and lead to

255 mutual promotion, and may also be the key to triggering a "cytokine storm" and

256 leading to critical illness and mortality. Pro-inflammatory neutrophils and C-reactive

257 protein may be one of the factors that make the cancer patients more serious and have

258 a poor prognosis after being infected with COVID-19.

259 Although we demonstrated the adverse effects of pro-inflammatory neutrophils and

260 CRP in cancer patients with COVID-19, our research still had several limitations.

261 First, this was a retrospective study, and the number of study cases is somewhat small.

262 There were also deficiencies in the number of medical records in the control group.

263 Second, we did not consider enough risk factors to establish a risk assessment model

264 and have not fully considered the unmeasured confounding factors. In the end, the

265 mechanism by which pro-inflammatory neutrophils and CRP aggravate the disease of

266 COVID-19 and cause poor prognosis remains to be further investigated. 
268 In summary, we presented some clinical features, laboratory data and outcomes of

269 cancer patients after being infected with COVID-19. Pro-inflammatory neutrophils

270 and CRP can be used as independent predictors of the severity and the adverse

271 clinical outcomes of COVID-19. Moreover, compared with COVID-19 patients

272 without cancer, cancer patients with COVID-19 have higher neutrophil counts and

273 CRP levels. Therefore, pro-inflammatory neutrophils and CRP play a greater adverse

274 role in patients with COVID-19 cancer, as a mediator to accelerate the interaction of

275 cancer and COVID-19, and promote the occurrence of "cytokine storm", which may

276 be COVID-19 causes of critical illness and adverse clinical outcomes in cancer

277 patients with COVID-19. During the outbreak of COVID-19, wo need to be more

278 alert to the levels of pro-inflammatory neutrophils and CRP after cancer patients

279 infected with COVID-19, so as to better give corresponding treatment measures.

281 Abbreviations

282 CRP: C-reactive protein; CK-MB: Creatine kinase-MB; IL: Interleukin; LDH: Lactate

283 dehydrogenase. 
Ethics approval and consent to participate

285 This study was reviewed and approved by Ethics Committee of the Tongji Medical

286 College of Huazhong University of Science and Technology (No.TJ-2020S098). The

287 exemption from informed patients' consent was approved by the ethics committee

288 because of the rapid spread of the infection and the rapid progression of some cases.

289 Consent for publication

290 Not applicable.

291 Availability of data and materials

292 Not applicable.

293 Competing interests

294 The authors declare that they have no competing interests.

$295 \quad$ Funding

296 This work was supported by the National Natural Science Foundation of China

297 [81773197 to B. Zhang]; Nature Science Foundation of Hubei Province

298 [2017CFB412 to J. Chen, 2017CFB633 to Y. Li]; and the joint research foundation of

299 Union Hospital in 2016. 
301 YL and JC designed the study and take responsibility for the integrity of the data and

302 the accuracy of the data analysis. BZ and YY interpreted the results and wrote the

303 manuscript. YY performed the analysis. SH substantially revised it. YZ, JL, SL, FX,

304 LZ, XL and WD collected and analyzed the data. All authors read and approved the

305 final manuscript.

\section{Acknowledgments}

307 Not applicable.

308 References

309 1. Zhu N, Zhang D, Wang W, Li X, Yang B, Song J, et al. A Novel Coronavirus

310 from Patients with Pneumonia in China, 2019. The New England journal of medicine.

$311 \quad 2020 ; 382(8): 727-33$.

312 2. Wu Z, McGoogan JM. Characteristics of and Important Lessons From the

313 Coronavirus Disease 2019 (COVID-19) Outbreak in China: Summary of a Report of

31472314 Cases From the Chinese Center for Disease Control and Prevention. Jama.

$315 \quad 2020$. 
316 3. Wang D, Hu B, Hu C, Zhu F, Liu X, Zhang J, et al. Clinical Characteristics of

317138 Hospitalized Patients With 2019 Novel Coronavirus-Infected Pneumonia in

318 Wuhan, China. Jama. 2020.

319 4. Li Q, Guan X, Wu P, Wang X, Zhou L, Tong Y, et al. Early Transmission

320 Dynamics in Wuhan, China, of Novel Coronavirus-Infected Pneumonia. The New

321 England journal of medicine. 2020;382(13):1199-207.

322 5. Li W, Zhang B, Lu J, Liu S, Chang Z, Cao P, et al. The characteristics of

323 household transmission of COVID-19. Clinical infectious diseases : an official

324 publication of the Infectious Diseases Society of America. 2020.

325 6. Chen W, Zheng R, Baade PD, Zhang S, Zeng H, Bray F, et al. Cancer statistics in

326 China, 2015. CA: a cancer journal for clinicians. 2016;66(2):115-32.

327 7. Siegel RL, Miller KD, Jemal A. Cancer statistics, 2019. CA: a cancer journal for 328 clinicians. 2019;69(1):7-34.

329 8. Liang W, Guan W, Chen R, Wang W, Li J, Xu K, et al. Cancer patients in SARS-

330 CoV-2 infection: a nationwide analysis in China. The Lancet Oncology.

$331 \quad 2020 ; 21(3): 335-7$. 
333 of COVID-19-infected cancer patients: a retrospective case study in three hospitals

334 within Wuhan, China. Annals of oncology : official journal of the European Society

335 for Medical Oncology. 2020.

336 10. Xia Y, Jin R, Zhao J, Li W, Shen H. Risk of COVID-19 for patients with cancer.

337 The Lancet Oncology. 2020;21(4):e180.

338 11. Wang H, Zhang L. Risk of COVID-19 for patients with cancer. The Lancet

339 Oncology. 2020;21(4):e181.

340 12. Luo X, Zhou W, Yan X, Guo T, Wang B, Xia H, et al. Prognostic value of C-

341 reactive protein in patients with COVID-19. Clinical infectious diseases : an official

342 publication of the Infectious Diseases Society of America. 2020.

343 13. Liu T, Zhang J, Yang Y, Ma H, Li Z, Zhang J, et al. The role of interleukin-6 in

344 monitoring severe case of coronavirus disease 2019. EMBO molecular medicine.

$345 \quad 2020$.

346 14. Moore JB, June CH. Cytokine release syndrome in severe COVID-19. Science.

347 2020;368(6490):473-4. 
349 clinical characteristics of 99 cases of 2019 novel coronavirus pneumonia in Wuhan,

350 China: a descriptive study. Lancet (London, England). 2020;395(10223):507-13.

351 16. Liu F, Li L, Xu M, Wu J, Luo D, Zhu Y, et al. Prognostic value of interleukin-6,

352 C-reactive protein, and procalcitonin in patients with COVID-19. Journal of clinical

353 virology : the official publication of the Pan American Society for Clinical Virology.

$354 \quad 2020 ; 127: 104370$.

355 17. Qin C, Zhou L, Hu Z, Zhang S, Yang S, Tao Y, et al. Dysregulation of immune

356 response in patients with COVID-19 in Wuhan, China. Clinical infectious diseases :

357 an official publication of the Infectious Diseases Society of America. 2020.

358 18. Mehta V, Goel S, Kabarriti R, Cole D, Goldfinger M, Acuna-Villaorduna A, et al.

359 Case Fatality Rate of Cancer Patients with COVID-19 in a New York Hospital

$360 \quad$ System. Cancer discovery. 2020.

361 19. Dai M, Liu D, Liu M, Zhou F, Li G, Chen Z, et al. Patients with Cancer Appear

362 More Vulnerable to SARS-CoV-2: A Multicenter Study during the COVID-19

363 Outbreak. Cancer discovery. 2020;10(6):783-91. 
20. Yang K, Sheng Y, Huang C, Jin Y, Xiong N, Jiang K, et al. Clinical

365 characteristics, outcomes, and risk factors for mortality in patients with cancer and 366 COVID-19 in Hubei, China: a multicentre, retrospective, cohort study. The Lancet 367 Oncology. 2020.

21. Greten FR, Grivennikov SI. Inflammation and Cancer: Triggers, Mechanisms,

369 and Consequences. Immunity. 2019;51(1):27-41.

22. Albrengues J, Shields MA, Ng D, Park CG, Ambrico A, Poindexter ME, et al.

371 Neutrophil extracellular traps produced during inflammation awaken dormant cancer

372 cells in mice. Science. 2018;361(6409).

373 23. Allin KH, Nordestgaard BG. Elevated C-reactive protein in the diagnosis,

374 prognosis, and cause of cancer. Critical reviews in clinical laboratory sciences.

$375 \quad 2011 ; 48(4): 155-70$.

376 24. Barnes BJ, Adrover JM, Baxter-Stoltzfus A, Borczuk A, Cools-Lartigue J,

377 Crawford JM, et al. Targeting potential drivers of COVID-19: Neutrophil

378 extracellular traps. The Journal of experimental medicine. 2020;217(6). 
380 Figure legend. Figure 1. Dynamic changes of inflammation-related indicators. A.

381 Dynamic changes of neutrophil counts in surviving patients and non-surviving patients

382 with COVID-19. B. Dynamic changes of C-reactive protein levels in surviving patients

383 and non-surviving patients with COVID-19.

384

385

386

387

388

389

390

Table 1. Univariate analysis of the correlation between laboratory parameters and severity of cancer patients with COVID-19

\begin{tabular}{lcccc}
\hline Laboratory Parameters & Moderate $(\mathrm{N}=14)$ & Severe $(\mathrm{N}=58)$ & Critical $(\mathrm{N}=40)$ & $P$ Value \\
\hline Erythrocyte count, $\times 10^{12} / \mathrm{L}$ & $3.99 \pm 0.55$ & $3.88 \pm 0.60$ & $3.12 \pm 0.94$ & $<0.001^{\#}$ \\
Hemoglobin count, $\mathrm{g} / \mathrm{L}$ & $123.93 \pm 12.06$ & $121.44 \pm 17.69$ & $93.00 \pm 27.42$ & $<0.001^{\#}$ \\
Platelet count, $\times 10^{9} / \mathrm{L}$ & $213.79 \pm 70.00$ & $219.29 \pm 91.78$ & $181.80 \pm 134.17$ & $0.235^{\#}$ \\
White blood cell count, $\times 10^{\prime} / \mathrm{L}$ & $4.39(3.92-6.32)$ & $5.88(4.80-8.20)$ & $6.49(4.67-10.70)$ & $0.083^{*}$ \\
Lymphocyte count, $\times 10^{9} / \mathrm{L}$ & $1.32 \pm 0.44$ & $1.41 \pm 1.73$ & $7.09 \pm 27.18$ & $0.228^{*}$ \\
Neutrophil count, $\times 10^{9} / \mathrm{L}$ & $2.71(2.17-4.06)$ & $3.95(2.91-5.83)$ & $4.88(2.71-8.28)$ & $0.031^{*}$ \\
Monocyte count, $\times 10^{\circ} / \mathrm{L}$ & $0.44 \pm 0.17$ & $0.53 \pm 0.27$ & $0.75 \pm 2.24$ & $0.668^{\#}$ \\
Aspartate aminotransferase, $\mathrm{U} / \mathrm{L}$ & $27.87 \pm 9.68$ & $38.40 \pm 26.57$ & $65.76 \pm 104.82$ & $0.076^{\#}$ \\
Alanine aminotransferase, $\mathrm{U} / \mathrm{L}$ & $29.38 \pm 14.93$ & $35.64 \pm 24.31$ & $36.49 \pm 38.48$ & $0.73^{\#}$
\end{tabular}




\begin{tabular}{|c|c|c|c|c|}
\hline Alkaline phosphatase, U/L & $66.79 \pm 23.33$ & $94.98 \pm 63.76$ & $245.43 \pm 565.63$ & $0.078^{\#}$ \\
\hline Creatinine, $\mu \mathrm{mol} / \mathrm{L}$ & $62.89 \pm 13.89$ & $85.40 \pm 107.09$ & $79.92 \pm 54.12$ & $0.665 \#$ \\
\hline Creatine kinase, $\mathrm{U} / \mathrm{L}$ & $64.00(48.50-92.15)$ & $62.00(40.00-87.00)$ & $63.00(30.00-108.00)$ & $0.903^{*}$ \\
\hline Lactate dehydrogenase, U/L & $193.22 \pm 40.96$ & $260.58 \pm 107.11$ & $451.86 \pm 447.58$ & $0.001^{\#}$ \\
\hline CK-MB, ng/ml & $0.83 \pm 0.51$ & $0.76 \pm 0.52$ & $2.18 \pm 3.02$ & $0.004^{\#}$ \\
\hline Troponin I, ng/L & $2.53 \pm 1.91$ & $8.83 \pm 15.55$ & $38.97 \pm 93.84$ & $0.041^{\#}$ \\
\hline Fibrinogen, g/l & $3.56 \pm 1.06$ & $4.45 \pm 1.41$ & $4.35 \pm 1.58$ & $0.123^{\#}$ \\
\hline D-dimer, mg/L & $0.53 \pm 0.47$ & $1.67 \pm 2.26$ & $3.75 \pm 4.96$ & $0.003^{\#}$ \\
\hline Procalcitonin, $\mu \mathrm{g} / \mathrm{L}$ & $0.04(0.01-0.13)$ & $0.06(0.03-0.25)$ & $0.60(0.22-1.40)$ & $<0.001^{*}$ \\
\hline $\mathrm{CRP}, \mathrm{mg} / \mathrm{L}$ & $0.78(0.28-11.68)$ & $24.10(4.90-55.95)$ & $82.33(44.81-124.50)$ & $<0.001^{*}$ \\
\hline $\mathrm{IL}-6, \mathrm{pg} / \mathrm{ml}$ & $6.42(3.81-11.00)$ & $10.05(4.45-29.51)$ & $32.79(10.51-53.41)$ & $<0.001^{*}$ \\
\hline TNF- $\alpha, p g / m l$ & $4.62 \pm 3.51$ & $6.05 \pm 11.51$ & $3.19 \pm 3.66$ & $0.399^{\#}$ \\
\hline $\mathrm{IL}-4, \mathrm{pg} / \mathrm{ml}$ & $2.27 \pm 0.78$ & $3.50 \pm 5.35$ & $2.46 \pm 1.45$ & $0.486^{\#}$ \\
\hline $\mathrm{IL}-2, \mathrm{pg} / \mathrm{ml}$ & $2.67 \pm 0.40$ & $3.03 \pm 0.90$ & $2.86 \pm 1.23$ & $0.591^{\#}$ \\
\hline $\mathrm{IL}-10, \mathrm{pg} / \mathrm{ml}$ & $3.30 \pm 0.83$ & $4.07 \pm 1.73$ & $6.19 \pm 3.97$ & $0.003^{\#}$ \\
\hline IFN- $\gamma, \mathrm{pg} / \mathrm{ml}$ & $2.09 \pm 0.66$ & $2.47 \pm 1.04$ & $3.08 \pm 3.73$ & $0.471^{\#}$ \\
\hline CD3 ratio, \% & $81.06 \pm 6.98$ & $66.42 \pm 20.46$ & $70.79 \pm 12.53$ & $0.082^{\#}$ \\
\hline CD4 ratio, \% & $50.87 \pm 4.41$ & $39.62 \pm 14.47$ & $37.40 \pm 14.69$ & $0.06^{\#}$ \\
\hline CD8 ratio, \% & $27.04 \pm 4.93$ & $23.69 \pm 12.91$ & $28.50 \pm 10.96$ & $0.253^{\#}$ \\
\hline CD4/CD8 ratio, \% & $1.93 \pm 0.34$ & $2.08 \pm 1.15$ & $1.53 \pm 0.96$ & $0.108^{\#}$ \\
\hline
\end{tabular}

Abbreviations: CRP, C-Reactive Protein; CKMB, Creatine Kinase-MB; $P<0.05$ was considered statistically significant. \# Indicates that the SK normality test follows the normal distribution, and expressed as mean \pm standard deviation. * Indicates that the SK normality test follows a nonnormal distribution, and expressed as median (interquartile range).

391

392

Table 2. Multivariate analysis of the correlation between laboratory parameters and severity of cancer patients with COVID-19

\begin{tabular}{lllll}
\hline Laboratory Parameters & Regression coefficients & OR & $P$ Value & $95 \% \mathrm{Cl}$ \\
\hline
\end{tabular}




\begin{tabular}{llllc} 
Erythrocyte count, $\times 10^{12} / \mathrm{L}$ & 1.389 & 4.01 & 0.337 & $0.24-68.44$ \\
Hemoglobin count, $\mathrm{g} / \mathrm{L}$ & -0.116 & 0.89 & 0.022 & $0.81-0.98$ \\
Neutrophil count, $\times 109 / \mathrm{L}$ & 0.589 & 1.80 & 0.021 & $1.09-2.97$ \\
Lactate dehydrogenase, U/L & 0.003 & 1.00 & 0.224 & $0.998-1.007$ \\
CK-MB, ng/ml & 0.462 & 1.59 & 0.595 & $0.29-8.71$ \\
Troponin I, ng/L & 0.024 & 1.02 & 0.133 & $0.99-1.06$ \\
D-dimer, mg/L & -0.172 & 0.84 & 0.456 & $0.53-1.32$ \\
Procalcitonin, $\mu \mathrm{g} / \mathrm{L}$ & 0.034 & 1.03 & 0.831 & $0.76-1.41$ \\
CRP, $\mathrm{mg} / \mathrm{L}$ & 0.035 & 1.04 & 0.043 & $1.001-1.071$ \\
IL-6, pg/ml & 0.008 & 1.01 & 0.405 & $0.99-1.025$ \\
IL-10, pg/ml & 0.299 & 1.35 & 0.344 & $0.73-2.50$ \\
\hline
\end{tabular}

Abbreviations: CRP, C-Reactive Protein; CKMB, Creatine Kinase-MB; $P<0.05$ was considered statistically significant.

393

394

Table 3. Univariate analysis of the correlation between laboratory parameters and severity of cancer patients with COVID-19

\begin{tabular}{lccc}
\hline \multicolumn{1}{c}{ Laboratory Parameters } & Alive $(\mathrm{N}=94)$ & Death $(\mathrm{N}=18)$ & $P$ Value \\
\hline Erythrocyte count, $\times 10^{12} / \mathrm{L}$ & $3.69 \pm 0.77$ & $3.20 \pm 0.99$ & $0.02^{\#}$ \\
Hemoglobin count, $\mathrm{g} / \mathrm{L}$ & $113.63 \pm 23.66$ & $99.67 \pm 31.06$ & $0.033^{\#}$ \\
Platelet count, $\times 10^{9} / \mathrm{L}$ & $210.19 \pm 102.75$ & $177.72 \pm 130.28$ & $0.245^{\#}$ \\
White blood cell count, $\times 10^{9} / \mathrm{L}$ & $5.53(4.30-7.85)$ & $8.94(5.94-12.26)$ & $0.003^{*}$ \\
Lymphocyte count, $\times 10^{9} / \mathrm{L}$ & $1.03(0.79-1.57)$ & $0.86(0.51-1.39)$ & $0.134^{*}$ \\
Neutrophil count, $\times 10^{9} / \mathrm{L}$ & $3.69(2.71-5.63)$ & $7.40(3.66-10.95)$ & $0.003^{*}$ \\
Monocyte count, $\times 10^{9} / \mathrm{L}$ & $0.42(0.31-0.62)$ & $0.41(0.20-0.65)$ & $0.542^{*}$ \\
Aspartate aminotransferase, $\mathrm{U} / \mathrm{L}$ & $29.00(20.00-45.00)$ & $35.50(21.25-76.00)$ & $0.428^{*}$ \\
Alanine aminotransferase, $\mathrm{U} / \mathrm{L}$ & $25.00(18.00-37.00)$ & $23.50(15.50-44.25)$ & $0.886^{*}$ \\
Alkaline phosphatase, $\mathrm{U} / \mathrm{L}$ & $74.00(61.00-104.00)$ & $103.00(76.00-288.50)$ & $0.01^{*}$ \\
Creatinine, $\mu$ mol/L & $65.00(53.68-79.48)$ & $64.10(54.18-106.68)$ & $0.556^{*}$
\end{tabular}




\begin{tabular}{|c|c|c|c|}
\hline Creatine kinase, U/L & $63.00(40.50-88.50)$ & $64.50(42.25-170.25)$ & $0.608^{*}$ \\
\hline Lactate dehydrogenase, U/L & $222.50(183.78-298.25)$ & 460.55 (223.75-639.25) & $0.001^{*}$ \\
\hline $\mathrm{CK}-\mathrm{MB}, \mathrm{ng} / \mathrm{ml}$ & $0.70(0.40-1.10)$ & $1.35(0.58-2.52)$ & $0.016^{*}$ \\
\hline Troponin I, ng/L & $3.80(1.90-10.25)$ & $14.10(6.30-39.38)$ & $0.003^{*}$ \\
\hline Fibrinogen, g/l & $4.28 \pm 1.43$ & $4.38 \pm 1.62$ & $0.788^{\#}$ \\
\hline D-dimer, mg/L & $0.90(0.24-1.84)$ & $3.18(0.01-6.57)$ & $0.324^{*}$ \\
\hline Procalcitonin, $\mu \mathrm{g} / \mathrm{L}$ & $0.09(0.03-0.30)$ & $0.61(0.36-2.52)$ & $<0.001^{*}$ \\
\hline $\mathrm{CRP}, \mathrm{mg} / \mathrm{L}$ & $24.10(3.79-61.60)$ & $93.02(77.45-182.25)$ & $<0.001^{*}$ \\
\hline IL-6, pg/ml & $10.10(5.42-32.68)$ & $39.18(24.70-89.36)$ & $0.001^{*}$ \\
\hline TNF- $\alpha, p g / m l$ & $2.69(2.10-3.58)$ & $2.26(1.50-2.84)$ & $0.09^{*}$ \\
\hline $\mathrm{IL}-4, \mathrm{pg} / \mathrm{ml}$ & $2.27(1.63-3.34)$ & $2.10(1.40-2.77)$ & $0.405^{*}$ \\
\hline $\mathrm{IL}-2, \mathrm{pg} / \mathrm{ml}$ & $2.69(2.39-3.53)$ & $2.59(2.18-3.27)$ & $0.521^{*}$ \\
\hline $\mathrm{IL}-10, \mathrm{pg} / \mathrm{ml}$ & $4.01(2.70-5.38)$ & $5.34(3.32-8.98)$ & $0.09^{*}$ \\
\hline IFN-y, pg/ml & $2.19(1.63-3.29)$ & $1.78(1.38-2.83)$ & $0.129^{*}$ \\
\hline CD3 ratio, \% & $69.90 \pm 17.82$ & $68.19 \pm 14.08$ & $0.764^{\#}$ \\
\hline CD4 ratio, \% & $40.54 \pm 14.13$ & $37.15 \pm 15.28$ & $0.471^{\#}$ \\
\hline CD8 ratio, \% & $26.27 \pm 12.16$ & $23.38 \pm 8.64$ & $0.453^{\#}$ \\
\hline CD4/CD8 ratio, $\%$ & $1.88 \pm 1.03$ & $1.75 \pm 1.16$ & $0.705^{\#}$ \\
\hline
\end{tabular}

Abbreviations: CRP, C-Reactive Protein; CKMB, Creatine Kinase-MB; $P<0.05$ was considered statistically significant. \# Indicates that the SK normality test follows the normal distribution, and expressed as mean \pm standard deviation. * Indicates that the SK normality test follows a non-normal distribution, and expressed as median (interquartile range).

395

396

Table 4. Multivariate analysis of the correlation between laboratory parameters and clinical adverse outcomes in cancer patients with COVID-19

\begin{tabular}{ccccc}
\hline Clinical Factor & Regression coefficients & OR & $P$ Value & $95 \% \mathrm{Cl}$ \\
\hline Erythrocyte count, $\times 10^{12} / \mathrm{L}$ & -0.86 & 0.423 & 0.704 & $0.005-35.822$ \\
Hemoglobin count, $\mathrm{g} / \mathrm{L}$ & 0.33 & 1.034 & 0.656 & $0.894-1.196$ \\
Neutrophil count, $\times 10^{9} / \mathrm{L}$ & 0.252 & 1.286 & 0.005 & $1.007-1.536$
\end{tabular}




$\begin{array}{ccccc}\text { Lactate dehydrogenase, U/L } & 0.002 & 1.002 & 0.104 & 1.000-1.004 \\ \text { Procalcitonin, } \mu g / L & -0.056 & 0.945 & 0.319 & 0.847-1.056 \\ \text { CRP, mg/L } & 0.046 & 1.047 & 0.003 & 1.016-1.079 \\ \text { IL-6, pg/ml } & 0.011 & 1.011 & 0.153 & 0.996-1.026\end{array}$

Abbreviations: CRP, C-Reactive Protein; $\mathrm{P}<0.05$ was considered statistically significant.

A

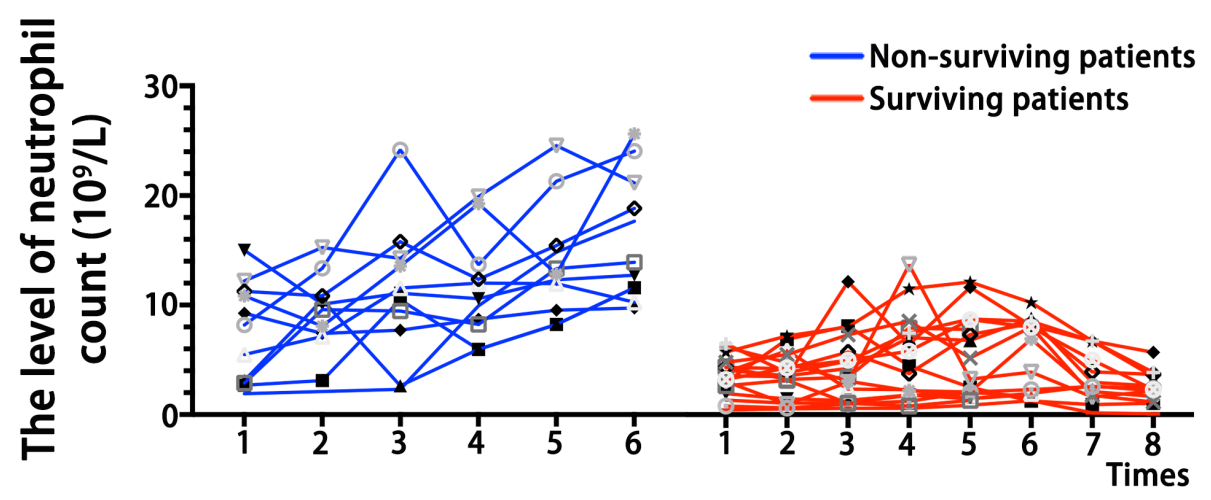

B

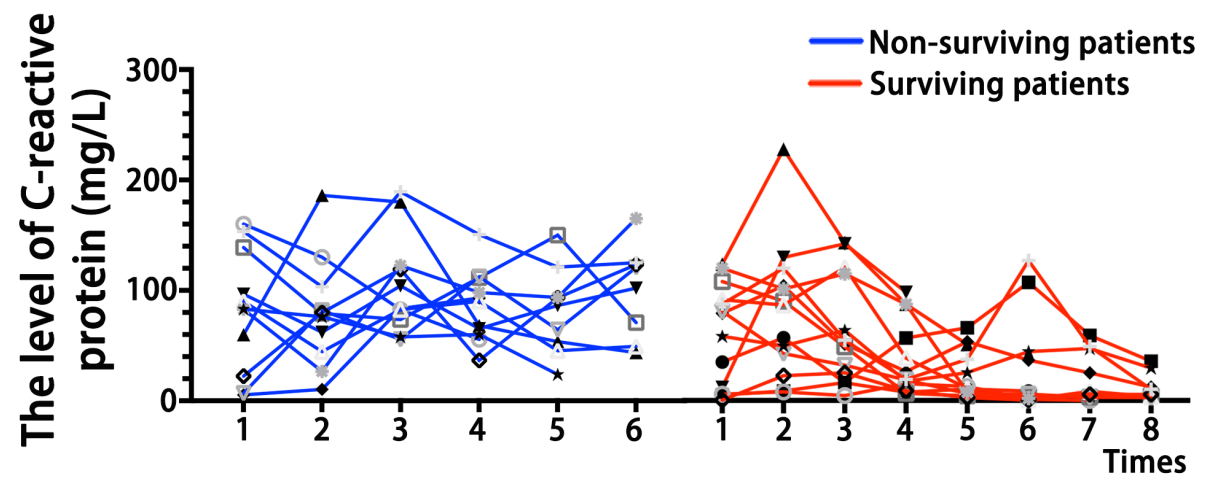

398 Figure 1. Dynamic changes of inflammation-related indicators. 


\section{Supplementary Files}

This is a list of supplementary files associated with this preprint. Click to download.

- Additionalfile1.pdf

- Additionalfile1.pdf 